\title{
ICOM \\ The land of rising science communication: the first Japan Scicom Forum
}

Conference

Reviewed by

Abstract

Keywords

DOI
JAPAN SCICOM FORUM, TOKYO, JAPAN, 20 APRIL 2018

\section{Ayumi Koso and Amanda Alvarez}

The first Japan Scicom Forum in Tokyo on April 20, 2018 gathered nearly 120 attendees to discuss the growing need and demand for English-language science communication in Japan and Asia. Keynotes and workshops addressed both the philosophy and motivations for scicomm in Japan and also the best practices for international outreach. Global science communication has reached a critical mass in Japan but securing sustainable funding, integrating the community and retaining momentum present ongoing challenges. As an online community and (hopefully) a recurring event, Japan Scicom Forum will foster a network of science communicators, professionalize and legitimize the field and boost English-language science communication in a country where it is still nascent.

Popularization of science and technology; Professionalism, professional development and training in science communication; Science and media

https://doi.org/10.22323/2.17030601

The inaugural Japan Scicom Forum (JSF; japanscicom.github.io) in April 2018 brought together nearly 120 writers, journalists and researchers, all actively engaged in, or curious about, communicating research in English. As the Japanese research community has experienced a recent need for and boom in global communication, this was the first large-scale event with the goals of fostering community and bolstering the professional identity of the field as well as sharing perspectives on English-language communication in Japan and Asia. The one-day event was generously hosted by the Earth-Life Science Institute (ELSI) at the Tokyo Institute of Technology. The participants, about half of whom were non-Japanese, represented 44 organizations including major universities, research institutions, government agencies, funders and academic publishers. Video of the keynotes is available on the YouTube channel of EurekAlert! at bit.ly/2IbzfHM. Collected tweets from the day are archived here: http:/ /wke.lt/w/s/u7FnV. 
Modern science communication is a fairly recent phenomenon in Japan, in part motivated by the Japanese government's change in science and technology policy from a public understanding model to a public engagement model [Watanabe, 2017]. The policy change brought about a surge in outreach activities in the Japanese language beginning around 2005 [Kobayashi, 2007]. Likewise, the need and boom in English-language science communication has been facilitated by top-down government initiatives. This pivot is motivated by falling university rankings as well as other indicators that suggest Japan's position as a scientific powerhouse is slipping [Phillips, 2017]. Japan's share of the global academic research literature has declined since the 2000s and the rate of scientific output has slowed. While international co-authoring has increased worldwide, Japanese researchers have not kept up with the pace of overseas collaborations [Saka and Kuwahara, 2010; Saka and Kuwahara, 2011]. Declines in citation indices also reveal a downshift in Japanese research quality and dissemination [Armitage, 2018].

In response, during 2013 and 2014 Japan's Ministry of Education, Culture, Sports, Science and Technology (MEXT) initiated two 10-year funding programs ("The Top Global University Project", "Program for Promoting the Enhancement of Research Universities") to raise the research performance and competitiveness of select Japanese universities and research institutions. Within this framework, English-language science communication is seen as a vehicle to achieve research excellence, as a tool to boost the global visibility of Japanese science and to enable Japanese organizations to engage with a wider audience and attract potential collaborators, faculty, students and funders. However, the resulting demand for English-language science communication has left institutions grasping for expertise and strategy. With these developments, the time was ripe to convene a national-level conference to empower and leverage the skills of science communicators across the language divide, and Japan Scicom Forum was born.

Japanese researchers frequently cite accountability to Japanese taxpayers as an excuse to avoid doing science communication beyond their native language. This clearly is a strong and valid, though perhaps restrictive, motivator for many Japanese scientists to engage with the public, as professor Takao Someya of the University of Tokyo outlined in his keynote at JSF. Someya is motivated by the bigger picture of shared social problems like climate change, which require engaging with global audiences. He has therefore embraced outreach via international media and his research has been covered by Time, $\mathrm{CNN}$, and others. The benefits and opportunities of global media engagement, like reversing brain drain and connecting with international collaborators, may not be apparent to his fellow Japanese scientists, says Someya, especially if these activities are not institutionally mandated, would necessitate a dedicated budget, or carry even minimal risk.

In a second keynote, Juliana Chan, founder of Asian Scientist Magazine, highlighted the "Asia gap" — the dominance of Western science in international news coverage and the lack of public recognition of Asian scientists. The motivation for producing science communication in English, in this case, aligns with the MEXT targets of name recognition and higher rankings. Indeed, the issue this raises - whether science communication in English is merely a means to an end or a goal in itself - is one that resonated throughout Japan Scicom Forum and deserves further national consideration. 
Additionally, science communicators from eight institutions across Japan shared their experiences and challenges in five-minute flash talks. Heated discussion emerged from a brief observation that Japanese-language press releases are generally detailed, longer in length and technical compared with English-language press releases. These differences may reflect disparate media landscapes and differing needs of journalists and reporters as well as unequal access to primary scientific literature.

The afternoon was devoted to hands-on workshops on pitching to the media, open science, press release culture and social media. Brian Lin of EurekAlert! encouraged press and public information officers to transcend press releases by pitching directly to international reporters. Press releases, he said, are akin to shopping at Uniqlo, the ubiquitous Japanese casual clothing chain, while pitching is like haute couture, custom made for the person you are targeting - two different strategies for very different purposes and audiences (Figure 1). University of Southern California's Sarah Mojarad gave practical tips for social media, like being mindful of time zones to reach different parts of the world and leveraging social media management tools for scheduling posts. When managing social media across two or more languages, looser adaptive translations that retain meaning rather than structure tend to fare better than literal translations.

The emerging landscape of English-language science communication in Japan still features language barriers, staffing and resource inequalities and hierarchical obstacles. Bringing together people with varying language abilities has been a challenge for the community, because events, organizations, trainings and policies tend to segregate linguistically. In planning Japan Scicom Forum, we endeavored to make the website, announcements and conference program bilingual, with simultaneous interpretation during the morning sessions. These efforts resulted in a balanced turnout of both Japanese and non-Japanese participants and we believe conscious actions of inclusivity and diversity are important for improving science communication quality across the board in Japan. A discussion group on the Trellis social network aims to retain the momentum of the community and provide a platform for continued exchange of best practices and ideas.

Fostering a grassroots community for English-language science communication in Japan is one way to channel the top-down initiatives into sustainable activities while also supporting the goals of research excellence and competitiveness. The traditional short-term efforts to 'solve' two distinct but inter-linked problems - Japan's waning global visibility in science and the national lack of English competence - are no longer sufficient. We are hopeful that networks like Japan Scicom Forum will contribute to the ongoing push for internationalization in higher education and science and technology policy, especially as the eyes of the world turn to Tokyo for the 2020 Olympics. This may also be an occasion for Japan to leverage its world-famous pop culture, like mascots and animation, in the service of science. The final keynote speaker at JSF, Mary Voytek of NASA, suggested these cultural references may serve as a touchstone and springboard for a uniquely Japanese flavor of scicomm - so next time you try to explain time travel, try Doraemon in place of Dr. Who! 
slı.do

What are some keywords you are taking away from the event?

Join at

slido.com

\#JSF18

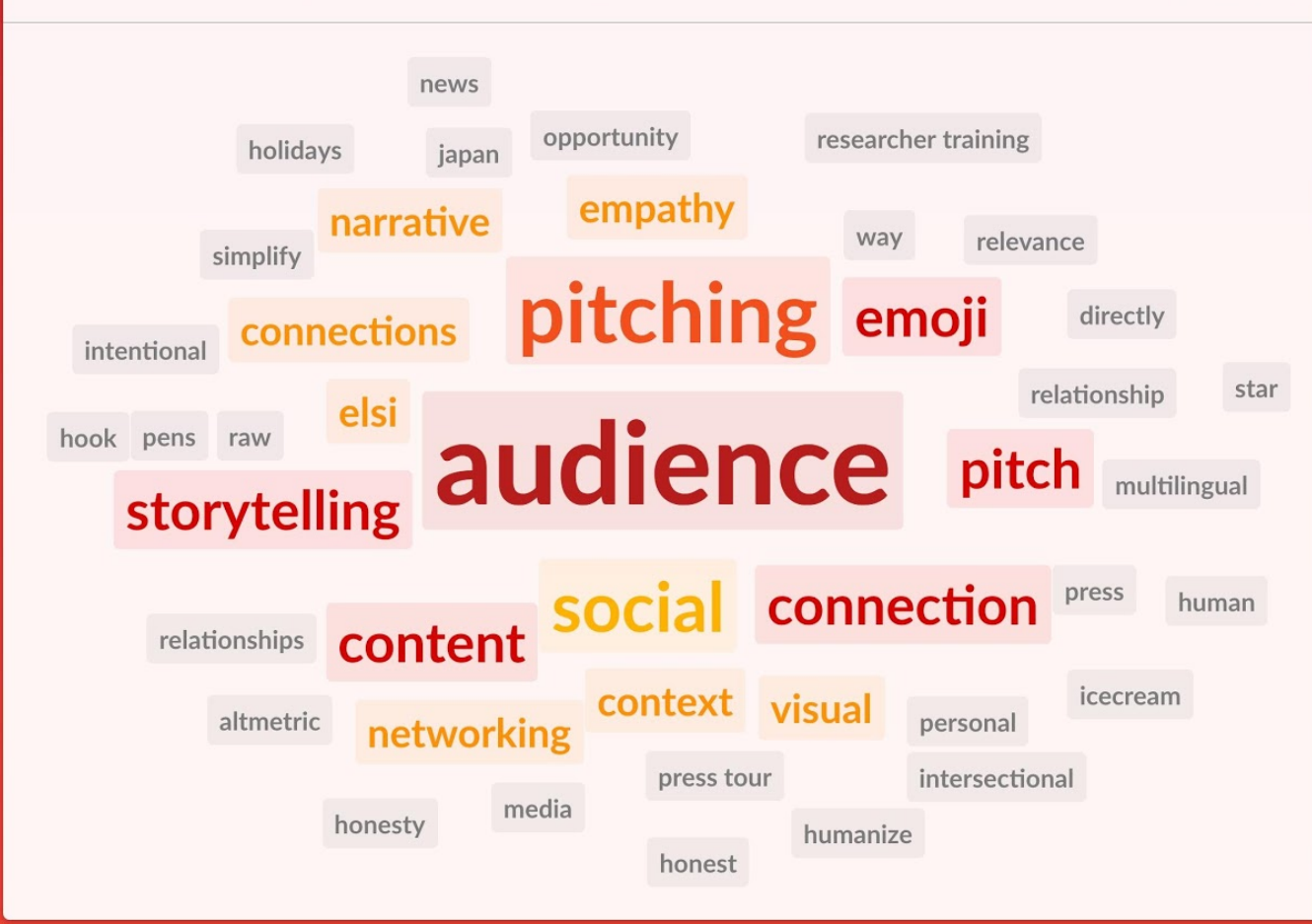

Figure 1. A word cloud with responses from 44 participants to the question "What are some keywords you are taking away from the event?". Word size and color saturation indicate more responses.
Acknowledgments

\section{References}

The authors thank Masataka Sasabe (RIKEN) for the Japanese translation of this paper.

Armitage, C. (2018). 'Stalled ambition'. Nature 555 (7697), S49-S49. https://doi.org/10.1038/d41586-018-02895-1.

Kobayashi, T. (2007). Toransu saiensu no jidai. [The age of trans-science]. Tokyo, Japan: NTT Publishing. ISBN: 978-4757160187.

Phillips, N. (23rd March 2017). 'The slow decline of Japanese research in 5 charts'. Nature Index. URL: https://www natureindex.com/news-blog/the-slow-decli ne-of-japanese-research-in-five-charts.

Saka, A. and Kuwahara, T. (2010). Benchmarking scientific research 2010 bibliometric analysis on dynamic alteration of research activity in the world and Japan. NISTEP Research Material 192. Japan: National Institute of Science and Technology Policy (NISTEP). URL: http://hdl . handle . net/11035/909.

- (2011). Benchmarking scientific research 2011 — bibliometric analysis on dynamic alteration of research activity in the world and Japan. NISTEP Research Material 204. Japan: National Institute of Science and Technology Policy (NISTEP). URL: http: //hdl . handle . net/11035/908.

Watanabe, M. (2017). 'From top-down to bottom-up: a short history of science communication policy in Japan'. JCOM 16 (03), Y01. URL: https://jcom.sissa.it/archive/16/03/JCOM_1603_2017_Y01. 
Ayumi Koso is project assistant professor at the National Institutes for the Humanities in Japan, an umbrella organization of six research organizations and museums, and visiting researcher at the National Bioscience Database Center, Japan Science and Technology Agency. She is also the co-organizer of Japan Scicom Forum. Her primary research areas are institution-level science/research communication in Japan and Asia. Ayumi received her Ph.D. from the Tokyo Metropolitan University and has worked as a public relations officer at the Japan Science and Technology Agency and the University of Tokyo. Twitter: @iyoumek. E-mail: koso@nihu.jp.

Amanda Alvarez is a science communicator at RIKEN, Japan's national science institute and the co-organizer of Japan Scicom Forum. She produces Nerd Nite Tokyo and before coming to Japan worked as a science writer in Switzerland, Germany and the United States. Amanda received her Ph.D. from the University of California, Berkeley. Twitter: @neuroamanda. E-mail: amanda.alvarez@riken.jp.

\section{How to cite}

Koso, A. and Alvarez, A. (2018). 'The land of rising science communication: the first Japan Scicom Forum'. JCOM 17 (03), R01. https: / / doi.org/10.22323/2.17030601. 